\title{
Extrahepatic portal venous obstruction: Is the knife irrelevant?
}

\author{
Sujoy Pal $\cdot$ Peush Sahni
}

A study on long term outcomes following variceal eradication in patients with extrahepatic portal venous obstruction (EHPVO) was clearly needed. In this issue of the Journal, Thomas et al. present the results of long-term follow up of a cohort of patients treated with endoscopic variceal sclerotherapy (EST). Of the 223 patients treated between 1985 and 1994, they had follow up data available for 198 patients. On a retrospective analysis of these data, 34 (17.2\%) patients had rebleeding after initial variceal eradication, 39 (19.7\%) had reappearance of esophageal varices and 19 (9.5\%) had de novo appearance of fundal varices. The mean duration from the time of eradication of varices to rebleeding was 5.4 (range 1 to 16) years. Based on these results, the authors suggest that endoscopic variceal eradication is an effective treatment modality for bleeding esophageal varices in patients with EHPVO.

This study, though retrospective, is quite thorough. ${ }^{1}$ It clarifies several questions, while throwing up some new ones. First, the authors base their premise on a cohort of patients in whom they had achieved successful initial hemostasis and long term variceal eradication. A large majority (87\%) of their patients presented with active variceal bleeding. However, we remain unsure of the outcome in patients in whom initial EST failed to achieve hemostasis. In $10 \%-15 \%$ of patients, endoscopic treatment may not achieve initial control of bleeding. ${ }^{2,3}$ This subset of patients is important since their exclusion may mislead us into

\section{S. Pal · P. Sahni}

Department of Gastrointestinal

Surgery and Liver Transplantation,

All India Institute of Medical Sciences,

Ansari Nagar,

New Delhi 110 029, India

P. Sahni $(\bowtie)$

e-mail: peush_sahni@hotmail.com

(C) Indian Society of Gastroenterology 2009 believing that EST is successful in all patients with variceal bleeding due to EHPVO. Secondly, 12 patients were lost to follow up from the initial cohort of 223 patients; did these patients die or undergo some other form of treatment elsewhere? No doubt the long term results are important, but without these initial data, establishing the overall efficacy of EST in this group of patients would be incomplete.

In the broader clinical perspective, the treatment options available for patients with EHPVO must include surgery. Evidence in the Western literature indicates that if shunt surgery has a role in treatment of portal hypertension, it is in patients with non-cirrhotic portal hypertension, and especially those with EHPVO. ${ }^{4,5}$ From India too, excellent long term results of proximal lieno-renal shunt (PLRS) have been reported in such patients. Of 160 children who underwent PLRS with splenectomy at our institution, the overall mortality was $1.9 \%$ (elective: $1 / 140$ [0.7\%]; emergency $2 / 20[10 \%]$ ), rebleeding rate was $11 \%$ and actuarial survival at 15 years by life-table analysis was $95 \% .{ }^{6,7}$ None of these patients developed encephalopathy. The procedure cured hypersplenism in all the patients who had this. Only one patient developed meningococcal meningitis, which recovered following treatment. Similarly, a study from Chandigarh that included 104 patients with non-cirrhotic portal hypertension who had undergone side-to-side lieno-renal shunt showed a shunt patency rate of $87 \%$, a rebleeding rate of $10 \%$, and no occurrence of encephalopathy after a mean follow up of 54 months. ${ }^{8}$ Thus, surgery is a one-time treatment procedure with durable, long term efficacy in preventing variceal rebleeding.

Long term studies (15-40 years) from the West have repeatedly shown that shunt surgery with or without splenectomy (proximal lieno-renal, side-to-side splenorenal, distal splenorenal or mesocaval) for EHPVO in children and adults, is associated with shunt patency rates between $90 \%$ and $95 \%$, rebleeding rates of $5 \%-10 \%$, no encephalopathy and 15-year actual survival rates of 95\%., ${ }^{5-11}$ These studies have found portosystemic shunts to be consistently effective for bleeding esophagogastric varices due to EHPVO. 
Some may argue that surgical option does not exist for the $10 \%$ of patients with EHPVO who have no shuntable vein or thrombosed spleno-portal and mesenterico-portal axis. Even in these situations, results of surgical esophagogastric devascularization have been encouraging. Mathur et al. reported a rebleeding rate of $11 \%$ and 5-year survival of $88 \%$ following a one-stage transabdominal esophagogastric devascularization with esophageal transection with or without splenectomy. ${ }^{12}$ Goyal et al. reported a rebleeding rate of $10 \%$ and a $95 \%$ overall survival among 22 patients with non-cirrhotic portal hypertension undergoing esophagogastric devascularization who were followed for a mean duration of 4 years. ${ }^{13}$ Orozco et al. from Mexico reported similar results in a selected group of patients with EHPVO $(n=38)$ undergoing the one-stage or two-stage Sugiura procedure. $^{14}$

The surgical options do not end here. A new type of shunt, the mesenterico-left portal vein bypass (Rex shunt), has been described recently, and is a new paradigm in surgical treatment. ${ }^{15}$ This procedure decompresses the portal venous system while simultaneously restoring portal blood flow to the liver. Follow up results ranging between 1 and 7 years are available. In the largest study reported till date, 34 patients underwent this procedure with a success rate of $91 \%$, good shunt patency rate, and rebleeding rate of $8 \%$ with amelioration of hypersplenism in most patients. ${ }^{15}$

Any therapeutic modality for management of variceal bleeding due to EHPVO has to be assessed against this long-term surgical experience. In addition, one must keep in mind that patients with EHPVO in India are young, have normal liver function, tolerate episodes of variceal bleeding well, and are generally expected to have a near normal life expectancy if bleeding does not recur.

Access to tertiary healthcare and blood bank facilities is still limited in large parts of India. It is in this context that the study published in this issue of the Journal assumes importance. This study has for the first time provided data based on a long duration of follow up after variceal obliteration of a large cohort of patients with EHPVO. ${ }^{1}$ It shows efficacy of EST by showing a high long term survival rate (195/198) and acceptable rebleeding rate $(\sim 17 \%)$. However, one has to also consider the potential for such therapy to adversely affect the natural course of the underlying disease, cause long term complications and alter the quality of life (QOL) of patients with EHPVO who are in the prime of their life.

It has been hypothesized that obliteration of varices by endoscopic means may induce the formation of new gastric varices, ectopic varices and severe portal hypertensive gastropathy, and may lead to worsening of portal biliopathy. $3,16,17$ This is thought to be due to the persistence of portal pressure. In the study under review, 10\% $(n=19)$ of patients developed new gastric varices, of whom 8 had rebleeding. ${ }^{1}$ A higher proportion of patients had severe portal hypertensive gastropathy on follow up and 3 developed ectopic duodenal varices. Of all the patients who had rebleeding $(n=34)$, about a quarter bled from gastric varices, 9\% from severe portal hypertensive gastropathy and $6 \%$ from ectopic varices. What is more important is that all these rebleeding events occurred in the late follow up period indicating that such sequelae may continue to evolve through the life of the patient. Although many of these rebleeding episodes were successfully managed using repeated sessions of sclerotherapy or endoscopic cyanoacrylate injection, and only one required surgery, one cannot discount the need for continued follow up after successful variceal eradication for period extending beyond 10 years and possibly even life long! Symptomatic portal biliopathy occurred in $12(6 \%)$ patients, of whom 4 required surgery; however, it is not known how many had biliopathy before sclerotherapy was initiated. Hence we cannot draw any inference about the natural course or progression of portal biliopathy from this study. We, as well as others have observed a high prevalence of asymptomatic biliopathy (84\%) in a cohort of patients with EHPVO who were followed up prospectively before and after PLRS with serial magnetic resonance cholangiopancreatography studies. ${ }^{18,19}$ Symptomatic portal biliopathy is a complex problem, which may have an unpredictable response to shunt surgery, may need repeated endoscopic biliary intervention procedures and surgical drainage, and may cause serious morbidity and mortality in the short as well as long term. ${ }^{20,21}$

QOL issues are particularly important in patients with EHPVO, who are usually young. A rigorous schedule of endoscopic variceal obliteration, and then follow up surveillance for 10-20 years, needs repeated hospital visits, which are likely to adversely impact a young patient and his/her family in many ways. In addition, about $15 \%$ of patients undergoing repeated EST may develop esophageal strictures requiring endoscopic dilatation ${ }^{1}$ adding to the hospital visits. It would be important to assess the overall impact of these demands on a growing child. Apart from the risk of rebleeding, these patients may have to contend with issues related to growth retardation ${ }^{22}$ and a large and at times painful spleen with persistent hypersplenism (about $20 \%$; primary indication for delayed surgery in $6 \%$ of cases in the study by Thomas et al. ${ }^{1}$

Surgical treatment, on the other hand, leads to a growth spurt, and reverses the hypersplenism and discomfort related to an enlarged spleen in all patients who undergo splenectomy and even many of those who do not undergo splenectomy. ${ }^{5-8}$ In a prospective study of patients with EHPVO undergoing shunt surgery, there was an overall improvement in scholastic abilities, physical activity and social interaction in a majority of the patients. ${ }^{23}$ Thus, the major benefit of surgical treatment is its being a one-time therapy that provides excellent long-term results and has the potential to beneficially modify the natural course of the disease. It is also cost-effective in the long run. 
Lest this sounds like an unabashed promotion of shunt surgery, we would like to emphasize that level 1 evidence is not available for any of the therapeutic modalities for patients with non-cirrhotic portal hypertension. The study by Thomas et al. in this issue of the Journal is an important contribution and provides long-term data on the role of endoscopic variceal eradication, that were hitherto not available. In India, the expertise to provide effective endoscopic variceal obliteration is more widely available than that for shunt surgery. Hence, despite several advantages of surgical therapy over endoscopic variceal obliteration, endoscopy will continue to be used as an effective modality to treat active variceal bleeding, and manage patients with failure of surgery as also varices in those patients who are not eligible for shunt surgery because of non-availability of a suitable vein. For the rest of the patients with EHPVO, endoscopists will continue to play a role till the expertise for surgery is more widely available. But the knife will remain relevant for times to come.

\section{References}

1. Thomas V, Jose T, Kumar S. Natural history of bleeding after esophageal variceal eradication in patients with extra hepatic portal venous obstruction: A twenty year follow-up. Indian J Gastroenterol 2009;28:206-11.

2. Bhargava DK, Dasarathy S, Atmakuri SP, Dwivedi M. Comparative efficacy of emergency endoscopic sclerotherapy for active variceal bleeding due to cirrhosis of the liver, non-cirrhotic portal fibrosis and extrahepatic portal venous obstruction. J Gastroenterol Hepatol 1990;5:432-7.

3. Sarin SK, Govil A, Jain AK, et al. Prospective randomized trial of endoscopic sclerotherapy versus variceal band ligation for esophageal varices: Influence on gastropathy, gastric varices and variceal recurrence. J Hepatol 1997;26:826-32.

4. Rikkers LF, Jin G, Langnas AN, Shaw BW Jr. Shunt surgery during the era of liver transplantation. Ann Surg 1997;226:51-7.

5. Orloff MJ, Orloff MS, Girard B, Orloff SL. Bleeding esophagogastric varices from extrahepatic portal hypertension: 40 years' experience with portal-systemic shunt. J Am Coll Surg 2002; 194:717-28.

6. Prasad AS, Gupta S, Kohli V, Pande GK, Sahni P, Nundy S. Proximal splenorenal shunts for extrahepatic portal venous obstruction in children. Ann Surg 1994;219:193-6.

7. Pande GK, Reddy VM, Kar P, et al. Operations for portal hypertension due to extrahepatic obstruction: results and 10 year follow up. Br Med J (Clin Res Ed) 1987;295:1115-7.

8. Mitra SK, Rao KL, Narasimhan KL, et al. Side-to-side lienorenal shunt without splenectomy in noncirrhotic portal hypertension in children. J Pediatr Surg 1993;28:398-401.
9. Bismuth H, Franco D, Alagille D. Portal diversion for portal hypertension in children: The first ninety patients. Ann Surg 1980;192:18-24.

10. Alvarez F, Bernard O, Brunelle F, Hadchouel P, Odièvre M, Alagille D. Portal obstruction in children. II. Results of surgical portosystemic shunts. J Pediatr 1983;103:703-7.

11. Orloff MJ, Orloff MS, Rambotti M. Treatment of bleeding esophagogastric varices due to extrahepatic portal hypertension: Results of portal-systemic shunts during 35 years. $J$ Pediatr Surg 1994;29:142-51.

12. Mathur SK, Shah SR, Nagral SS, Soonawala ZF. Transabdominal extensive esophagogastric devascularization with gastroesophageal stapling for management of noncirrhotic portal hypertension: long-term results. World J Surg 1999;23:1168-74.

13. Goyal N, Singhal D, Gupta S, Soin AS, Nundy S. Transabdominal gastroesophageal devascularization without transection for bleeding varices: Results and indicators of prognosis. J Gastroenterol Hepatol 2007;22:47-50.

14. Orozco H, Takahashi T, Mercado MA, Prado E, Chan C. Surgical management of extrahepatic portal hypertension and variceal bleeding. World J Surg 1994;18:246-50.

15. Superina R, Bambini DA, Lokar J, Rigsby C, Whitington PF. Correction of extrahepatic portal vein thrombosis by the mesenteric to left portal vein bypass. Ann Surg 2006;243:515-21.

16. Gonçalves ME, Cardoso SR, Maksoud JG. Prophylactic sclerotherapy in children with esophageal varices: long-term results of a controlled prospective randomized trial. J Pediatr Surg 2000;35:401-5.

17. Itha S, Yachha SK. Endoscopic outcome beyond esophageal variceal eradication in children with extrahepatic portal venous obstruction. J Pediatr Gastroenterol Nutr 2006;42:196-200.

18. Desai P. Role of MRCP in delineating biliary abnormalities in patients with EHO before and after proximal lienorenal shunt [MCh (GI Surgery) dissertation]. New Delhi: All India Institute of Medical Sciences; 2000.

19. Dhiman RK, Behera A, Chawla YK, Dilawari JB, Suri S. Portal hypertensive biliopathy. Gut 2007;56:1001-8.

20. Singh RK, Pal S, Sahni P, Acharya SK, Chattopadhyay TK. Obstructive jaundice due to portal biliopathy-our experience with shunt surgery. J Gastroenterol Hepatol 2004;19 (Suppl):A691.

21. Chaudhary A, Dhar P, Sarin SK, et al. Bile duct obstruction due to portal biliopathy in extrahepatic portal hypertension: surgical management. Br J Surg 1998;85:326-9.

22. Sarin SK, Bansal A, Sasan S, Nigam A. Portal-vein obstruction in children leads to growth retardation. Hepatology 1992;15:229-33.

23. Menon P, Rao KL, Bhattacharya A, et al. Extrahepatic portal hypertension: Quality of life and somatic growth after surgery. Eur J Pediatr Surg 2005;15:82-7. 